\title{
DIx genes and the maintenance of bone homeostasis and skeletal integrity
}

\author{
G Levi $^{\star * 1}$ and Y Gitton ${ }^{1}$ \\ Cell Death and Differentiation (2014) 21, 1345-1346; doi:10.1038/cdd.2014.94
}

Bone development and bone homeostasis $(\mathrm{BH})$ are highly integrated processes that support skeletal formation, maintenance and repair during the whole lifespan. $\mathrm{BH}$ depends on the concerted action of specialized cell populations responsible for either bone apposition (osteoblasts) or resorption (osteoclasts). Osteoblasts and osteoclasts coordinate their action through endocrine and paracrine soluble molecular signals. Similar molecular cues also affect the differentiation of mesenchymal and hematopoietic progenitors influencing the expression and activity of key osteogenic transcription factors. ${ }^{1}$

$\mathrm{BH}$ requires coordination of three major processes: (1) osteoblast differentiation and bone matrix production, (2) osteoblast-dependent regulation of osteoclastogenesis through the secretion of Receptor Activator of NF- $\kappa \mathrm{B}$ Ligand (RANKL) and osteoprotegerin (OPG ${ }^{2}$ ), and (3) cell-autonomous regulation of osteoclast activity. ${ }^{3}$

Defects in $\mathrm{BH}$ are at the origin of common and severe pathologies such as, for example, osteoporosis, a complex disease characterized by a reduction in bone mass with associated bone microarchitectural deterioration and a high risk of fractures. Although environmental factors such as nutrition and mechanical load, as well as lifestyle, may influence bone development and $\mathrm{BH}$, family and twin pair studies have suggested a strong genetic component for these processes.

Bone-related disorders are a major social and economic burden for any health-care system as they affect particularly the aging population, which is increasing in most societies. New insight into the transcriptional programs supporting $\mathrm{BH}$ and bone remodelling is, therefore, essential to tackle the challenge posed by the increasing incidence of bone disease.

Mammalian D/x homeobox transcription factors constitute a gene family formed by three bigenic clusters: $D / x 1$ and $D / x 2$; DIx5 and DIx6; DIx3 and DIx4. ${ }^{4-6}$ These six Dlx genes are differentially expressed by the cellular components of bone: chondrocytes, osteoblasts and osteoclasts. ${ }^{7}$ DIx transcription factors my well play different and synergic roles in the control of chondrogenesis and/or osteogenesis. ${ }^{8,9}$

In particular, mutations in $D L X 3$ result in Tricho-DentoOsseous syndrome, an autosomal dominant ectodermal dysplasia characterized by curly kinky hair, enamel hypoplasia, taurodontism and increased bone mineral density (BMD) in intramembranous and endochondral bones. ${ }^{10}$ The importance of DLX3 in bone is also supported by its capacity to regulate directly in vitro critical determinants of bone differentiation such as osteocalcin (Ocn), Runx2 and osteoactivin, ${ }^{11-13}$ and by the observation that overexpression of DLX3 in osteoprogenitors stimulates transcription of osteogenic markers.

Most in vivo functional studies on the skeletal regulatory function of $D / x$ genes have been performed either during embryonic development (e.g.: Samee et al. ${ }^{9}$ ) or in organ culture (e.g.: Hsu et al. ${ }^{14}$ ) as early lethality of null mutant mice has often hampered the elucidation of their postnatal role.

In this issue of Cell Death Differentiation, Isaac et al. ${ }^{15}$ perform the first in vivo study of the postnatal role of $D L X 3$ in the appendicular skeleton. They generate and analyse mice carrying conditional loss-of-function mutations of $D / x 3$ in mesenchymal cells $\left(D / x 3^{\text {Prx1-cKO }}\right)$ or in osteogenic lineage cells $\left(D / \times 3^{O C N-C K O}\right)$. Both models present a significant increase in bone mass throughout their lifespan. In the absence of DIx3 endochondral bone formation still takes place at the growth plate, but bone deposition is significantly higher, with trabecular bone extending into the medullary cavity. Cortical bone is thicker, but more porous and with decreased BMD.

By combining in vivo gene profiling and ex vivo cellular analyses, the authors conclude that the increase in trabecular bone mass observed in $D / \times 3^{O C N-C K O}$ mice does not arise from impaired osteoclastic activity but from direct enhancement of the bone-forming osteoblast activity with an imbalance in $\mathrm{BH}$ in favour of bone apposition. RNA-Seq analysis of $D / x 3^{O C N-C K O}$ metaphysis shows upregulation of transcription factors essential for osteoblastogenesis, including Run $x 2,{ }^{16}$ its downstream osteoblast-specific target $S p 7^{17}$ and $D / \times 5 / D / x 6$, two positive regulators of chondrocyte and osteoblast differentiation (see Figure 1). ${ }^{8,9,18,19}$

The fact that Dlx3 plays an important role in regulating osteoblast activity is further supported by the analysis of DIx3-deleted bone marrow stroma cells (BMSCs), which also shows increased osteoblast differentiation and bone-forming activity associated with increased gene expression of Runx2 and DIX5. The notion that DLX3 acts as a negative regulator of osteoblastogenesis by reducing Runx2, Sp7, DIx5 and DIx6 gene expression is further supported by ChIP analysis on

\footnotetext{
'Évolution des Régulations Endocriniennes, CNRS UMR7221, Muséum National d'Histoire Naturelle, Paris 75005, France

*Corresponding author: G Levi, Évolution des Régulations Endocriniennes, CNRS UMR7221, Muséum National d'Histoire Naturelle, 7 rue Cuvier, UMR7221 CNRS/MNHN, Paris Cedex 05 75005, France. Tel: +33 1 40793621; Fax: +33 1 40793618; E-mail: glevi@mnhn.fr
} 


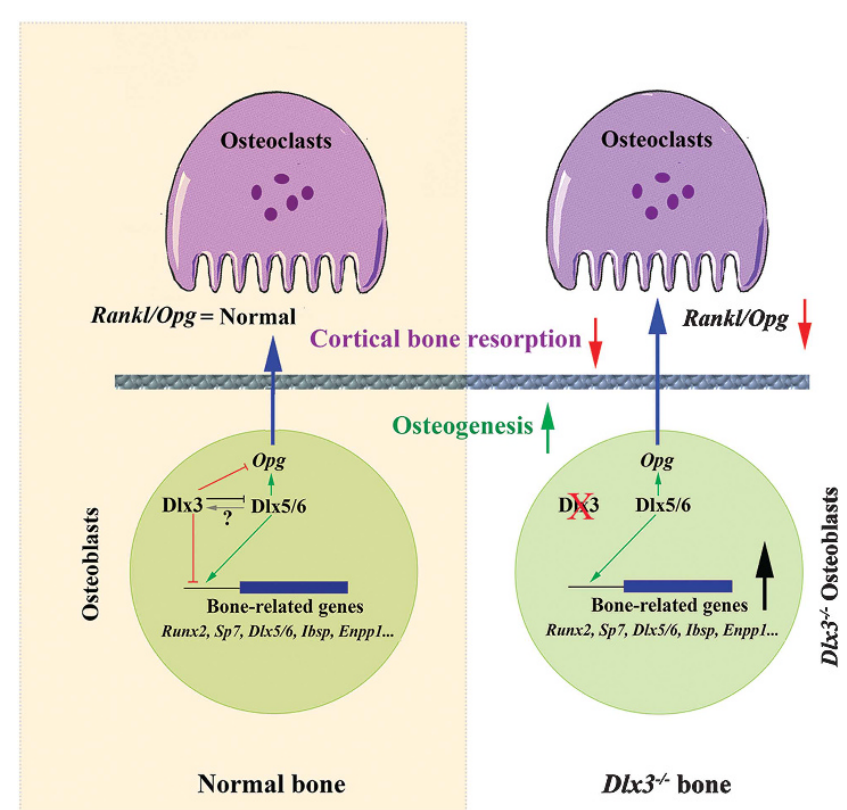

Figure 1 Summary diagram of the effects of $D / x 3$ inactivation in osteogenic cells. In osteoblast (lower part of the figure) DIx3 inhibits the expression of positive regulators of osteogenesis. DIx 3 inactivation results in higher levels of expression of Runx2, Sp7, Dlx5/6, and so on, and increases osteogenesis. Cortical bone osteobalsts of DIx3 - / - mice present also a higher level of expression of Opg, suggesting that, in parallel with increased bone deposition, this bone compartment is also characterized by reduced activity of osteoclasts (upper part of Figure 1). Dlx3 is, therefore, a negative modulator of osteogenesis at multiple levels; its coordinated action with other activators of osteogenesis, such as Dlx5, is required for the maintenance of bone homeostasis

BMSCs, which shows that DLX3 binds to the promoters of Sp7, DIx5 and DIx6 and Runx2, directly modulating their activity (see also Hassan et al. ${ }^{12}$ ).

An interesting concept that emerges from this study is that the combined action of DIx3 and DIx5/6 generates a regulatory network essential for the maintenance of $\mathrm{BH}$. First of all, the data (both in vivo and in vitro) indicate that DIx3 negatively regulates D/x5/6 in bone, whereas previous studies showed that the targeted simultaneous inactivation of $D / x 5$ and $D / x 6^{20}$ results in the loss of DIx3 expression. Taken together, these results suggest that bone formation and homeostasis implicate reciprocal regulatory loops between DIx5/DIx6 and DIx3. In vitro studies have shown that DIx3 and DIx5 associate with the Ocn promoter at the onset of transcriptional activation, concomitant with Runx2 occupancy, and, while DIx3 occupancy on the Ocn promoter decreases from osteoblast maturation to mineralization, Dlx5 occupancy is maximal during the mineralization stage. ${ }^{12}$

Isaac et al. ${ }^{15}$ demonstrate that $D / x 3$ deletion in calvaria osteoblasts results in increased occupancy of DIx5 and increased and premature occupancy of Runx2 on regulatory elements on the Ocn promoter. Thus, D/x3 deletion could directly affect the network of molecular switches and promote osteoblastic differentiation and bone-forming activity via an enhancement of the occupancy of bone-activator TFs such as DIx5 and Runx2. Therefore, Dlx3 and Dlx5 seem to have coordinated and opposite roles in the transcription of bone-related genes via molecular switches at their promoter regions.

Inactivation of $D / x 3$ results in an increased thickness of cortical bone associated with increased mineral apposition rate in the periosteum, decreased BMD, increased porosity and vascularization. RNA-Seq analysis of cortical bone of mutant mice revealed upregulation of Opg and Mepe, two major inhibitors of osteoclastogenesis strongly expressed by late-differentiated osteoblasts and osteocytes. ChIP analysis demonstrates that Dlx3 binds to several sites on the Opg promoter. These findings bolster the concept that $D / x 3$ inactivation in osteoblasts induces increased levels of Opg and Mepe in cortical bone, thereby deregulating homeostasis in favour of enhanced bone deposition (see Figure 1). Indeed, $D / \times 5^{-/-}$mice have an increased number of osteoclasts while $D / x 5^{-/-}$osteoblasts exhibit decreased Opg expression, resulting in a higher Rankl/Opg ratio. ${ }^{9}$ It seems plausible that, besides Dlx5, ${ }^{9}$ Dlx3 is also a central regulator of osteoblast/ osteoclast coupling. However, conversely to DIx5, Dlx3 would inhibit osteoblast bone-forming activity via a negative transcriptional control of genes responsible for bone formation, while simultaneously activating bone resorption through osteoblast-activated regulation of osteoclastogenesis. It seems therefore that, throughout life, DIx 3 is a negative modulator of osteogenesis and that its coordinated action with other TFs, such as DIx5, is required for the maintenance of $\mathrm{BH}$. DLX3 is therefore an interesting target for future translational research in human skeletal disorders such as osteopososis.

\section{Conflict of Interest}

The authors declare no conflict of interest.

1. Karsenty G et al. Annu Rev Cell Dev Biol 2009; 25: 629-648.

2. Boyle WJ et al. Nature 2003; 423: 337-342.

3. Negishi-Koga T, Takayanagi H. Immunol Rev 2009; 231: 241-256.

4. Cohen SM et al. Nature 1989; 338: 432-434.

5. O'Hara E et al. Development 1993; 117: 847-856.

6. Panganiban G, Rubenstein JL. Development 2002; 129: 4371-4386

7. Li H et al. Dev Biol 2008; 316: 458-470.

8. Samee N et al. Crit Rev Eukaryot Gene Expr 2007; 17: 173-186.

9. Samee $N$ et al. Am J Pathol 2008; 173: 773-780.

10. Haldeman RJ et al. Bone 2004; 35: 988-997.

11. Hassan MQ et al. Mol Cell Biol 2004; 24: 9248-9261.

12. Hassan $M Q$ et al. J Biol Chem 2006; 281: 40515-40526.

13. Singh $\mathrm{M}$ et al. J Cell Physiol 2012; 227: 390-399.

14. Hsu SH et al. Mech Dev 2006; 123: 819-830.

15. Isaac J et al. Cell Death Differ 2014; 21: 1365-1376.

16. Otto F et al. Cell 1997; 89: 765-771.

17. Nakashima $\mathrm{K}$ et al. Cell 2002; 108: 17-29.

18. Lee MH et al. J Biol Chem 2005; 280: 35579-35587.

19. Tadic T et al. J Bone Miner Res 2002; 17: 1008-1014

20. Depew MJ et al. Science 2002; 298: 381-385. 